\title{
Analysis of Self-Homodyne and Delayed Self-Heterodyne Detections for Tunable Laser Source Linewidth Measurements
}

\author{
Dr Adnan Hussein Ali ${ }^{1}$, Dr. Sahib N. Abdul-Wahid ${ }^{2}$ \\ ${ }^{1}$ Electrical \& Electronics Techniques College - Baghdad, Iraq \\ ${ }^{2}$ Kufa University, Najaf, Iraq
}

\begin{abstract}
The linewidth characteristic of a commercial tunable laser source (TLS) is experimentally demonstrated with two different linewidth measurement methods; homodyne and heterodyne detections. With $25 \mathrm{~km}$ of SMF as a delay fiber and wide mode operation, the linewidths of $124 \mathrm{MHz}, 114 \mathrm{MHz}$ and $176 \mathrm{MHz}$ are obtained at operating wavelengths of $1530 \mathrm{~nm}, 1540 \mathrm{~nm}$ and $1560 \mathrm{~nm}$, respectively. The linewidth of the TLS is obtained at around $10 \mathrm{MHz}$ and $150 \mathrm{MHz}$ for narrow and wide modes, respectively with heterodyne technique.
\end{abstract}

Key words: tunable laser source (TLS), Self-homodyne, Delayed self-heterodyne detections, and laser linewidth measurement

\section{Introduction}

Recently developed laser sources show improved coherence properties and linewidth in the submegahertz range will be available in a near future even in monolithic semiconductor lasers. The linewidth measurement of such lasers is a new challenge, since classical self-homodyne or self-heterodyne techniques require excessive lengths of delaying fibre and are thus not practicable.

The linewidth of a laser is the full width at half-maximum (FWHM) of its optical spectrum. The linewidth is strongly related to the temporal coherence, characterized by the coherence time or coherence length. A laser linewidth can be measured with a variety of techniques such as using optical spectral analysis as well as converting the frequency fluctuation into intensity fluctuation. For single-frequency lasers, the self-heterodyne technique is often used, which involves recording a beat note between the laser output and a frequency-shifted and delayed version of it. For sub-kilohertz linewidths, the ordinary self-heterodyne technique usually becomes impractical, but it can be extended by using a recirculating fiber loop with an internal fiber amplifier. Lasers with very narrow linewidth are required for various applications such as light sources for various kinds of fiberoptic sensors, for LIDAR spectroscopy, in coherent optical fiber communications, and for test and measurement purposes[1].

The linewidth of a laser depends strongly on the type of laser. It may be further minimized by optimizing the laser design and suppressing external noise influences as far as possible. The first step should be to determine whether spontaneous emission noise is dominating, because the required measures can depend very much on this. The influence of spontaneous emission noise is small for a laser with high intra-cavity power, low resonator losses, and a long resonator round-trip time. Single frequency fiber lasers can achieve linewidths of a few kilohertz, or sometimes even below $1 \mathrm{kHz}$. The linewidth of a laser diode is typically in the megahertz region, but it can also be reduced to a few kilohertz, e.g. in external-cavity diode lasers, particularly with optical feedback from a high-finesse reference cavity [2]. Small fiber lasers in the form of distributed feedback lasers (with the resonator formed essentially by a special fiber Bragg grating) can generate tens of mill watts of output power with a linewidth in the region of a few kilohertz.

The Brillouin gain in optical fibers can be used to make narrow linewidth lasers by placing a piece of highly nonlinear fiber inside a cavity. We can use the nonlinear Brillouin gain to produce Brillouin fiber laser, which can be generated at any wavelength depending on the availability of the Brillouin pump.

\section{A. Self-homodyne detection}

\section{II. linewidth measurement techniques}

In coherent detection, the frequency of the input optical signal is down converted to the RF domain through mixing with a local oscillator. Self-homodyne detection eliminates the requirement of a local oscillator, and the optical signal mixes with a delayed version of itself. Fig.1 shows the self-homodyne detection set-up, where two delay lines are used between two directional couplers to form a Mach-Zehnder configuration. 


\section{B. Heterodyne Detection}

Heterodyne detection can provide not only laser linewidth data, but also optical power spectrum. This method is the only technique that is capable of characterizing non symmetrical spectral lineshape. This method also offers high sensitivity and high resolution. The key component required for this method is a stable, narrow line-width reference laser. In heterodyne detection, two lasers have to be used. One is the signal laser and another one is a reference laser, which can be referred as the local oscillator (LO). The central frequency of the LO laser must be tuned close to the signal laser frequency to allow the mixing product to fall within the bandwidth of detection device.

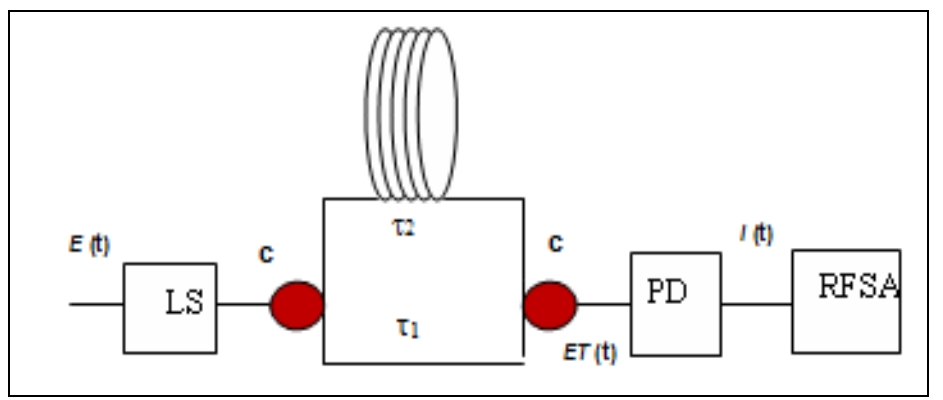

Fig. 1: Typical experimental set-up for linewidth measurement using self-homodyne method.

Fig. 2 shows the typical experimental set-up for heterodyne detection method whereby a light from the LO is combined with the signal laser under test by a $3 \mathrm{~dB}$ coupler. The coupler combines the two fields, delivering partial power to each output port. One port leads to a photodetector (PD) which detects the interference beat note, converting it to an electrical tone. The main difficulty to use heterodyne detection is that two lasers must be used and the linewidth of the reference laser must be narrower than or at least comparable to that of the laser source to be measured in order to achieve reasonable measurement accuracy[3] [4].

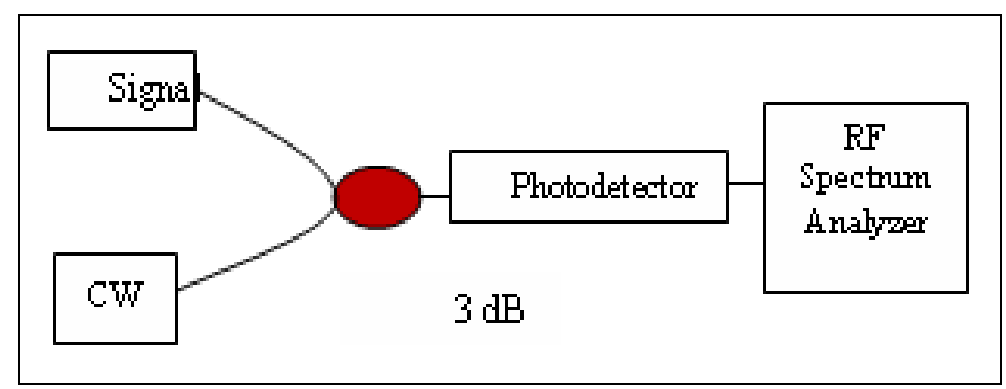

Fig.2 Typical experimental set-up for linewidth measurement using a heterodyne beat technique.

\section{Delayed self-heterodyne detection}

Compared with heterodyne detection, delayed self-heterodyne detection provides a simpler method to perform laser linewidth measurement without using a separate local oscillator. Instead, delayed self-heterodyne detection needs a large optical delay. The delayed self-heterodyne interferometer (DSHI) concept is shown in Fig. 3. Incident light is split into two paths by the interferometer. The optical frequency of one arm is offset with respect to the other. If the delay, $\tau_{d}$, of one path exceeds the coherence time, $\tau_{c}$ of the source, the two combining beams interfere as if they originated from two independent lasers offset in frequency by $\delta v$. Thus the system performs similarly to optical heterodyne detection.

\section{Self- homodyne linewidth measurement:}

A linewidth measurement for a commercial tunable laser source (TLS) is demonstrated in this chapter. Fig. 4 shows the experimental set-up, which consists of TLS (ANDO AQ4321D), 1 × $23 \mathrm{~dB}$ coupler, a piece of long SMF $(25 \mathrm{~km} \& 50 \mathrm{~km})$ length, photo-detector, and $8 \mathrm{GHz}$ radio frequency spectrum analyzer. The input light from TLS is split by the first $3 \mathrm{~dB}$ coupler, which one arm is connected to the second $3 \mathrm{~dB}$ coupler. Another arm of the coupler is connected to a piece of delay SMF connected to the second $3 \mathrm{~dB}$ coupler. The combined light at second coupler is converted into electrical signal by a photodiode, which the signal is analyzed by the RFSA.

Fig. 5 shows the output spectrum of TLS at power setting of $0 \mathrm{dBm}$, linewidth setting of 'wide' and wavelength of $1550 \mathrm{~nm}[5]$. 


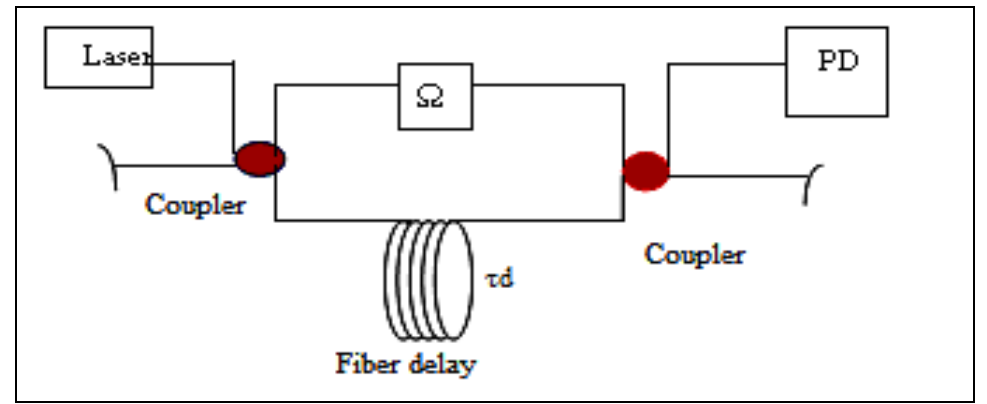

Fig. 3: Schematic setup for optical delayed self-heterodyne detection

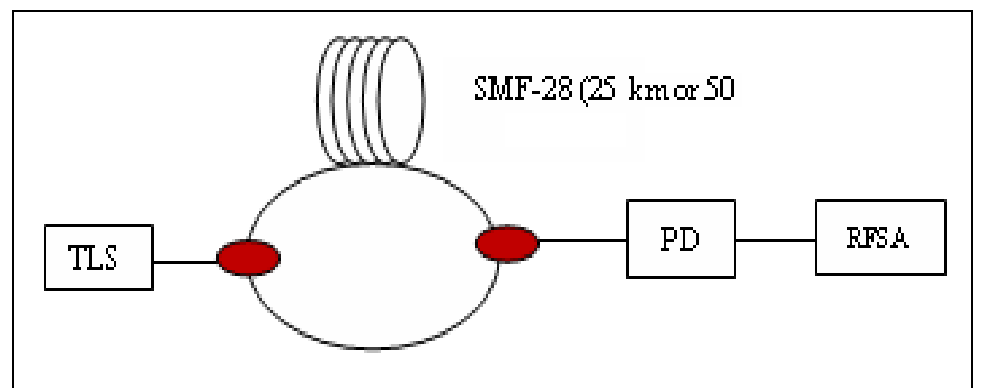

Fig. 4: Experimental set-up for self-homodyne linewidth measurement of a TLS

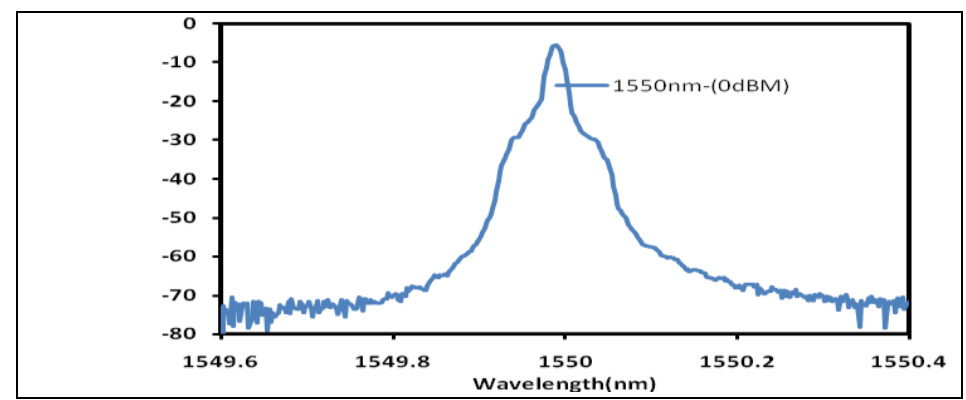

Fig. 5: The spectrum of the input signal from TLS

Fig. 6 shows the beat frequency spectrum at three different operating wavelengths. In the experiment, the TLS power and SMF length is fixed at $0 \mathrm{dBm}$ and $25 \mathrm{~km}$, respectively. The linewidths of $124 \mathrm{MHz}, 114 \mathrm{MHz}$ and $176 \mathrm{MHz}$ are obtained at operating wavelengths of $1530 \mathrm{~nm}, 1540 \mathrm{~nm}$ and $1560 \mathrm{~nm}$, respectively. The values of the linewidth are obtained at $3 \mathrm{~dB}$ from the peak signal from the graphs in Fig. 6 . Fig 7 shows the beat frequency spectrum at three different operating wavelengths but using a longer delay fiber of $50 \mathrm{~km}$. The linewidths of 320 $\mathrm{MHz}, 120 \mathrm{MHz}$ and $194 \mathrm{MHz}$ are obtained at operating wavelengths of $1530 \mathrm{~nm}, 1540 \mathrm{~nm}$ and $1560 \mathrm{~nm}$, respectively. It is found that a slightly higher value is obtained with a longer length of the delay fiber.

Fig. 8 shows the linewidth of light against wavelength of the TLS at two different delay fibers. In the experiment, the TLS option is set as 'wide' mode. As shown in the figure, the average linewidth of the TLS is around $200 \mathrm{MHz}$. Since the result for $25 \mathrm{~km}$ and $50 \mathrm{~km}$ delay fibers are almost similar, it is concluded that 25 $\mathrm{km}$ long of SMF is enough of provide delay for the self-homodyne linewidth measurement.

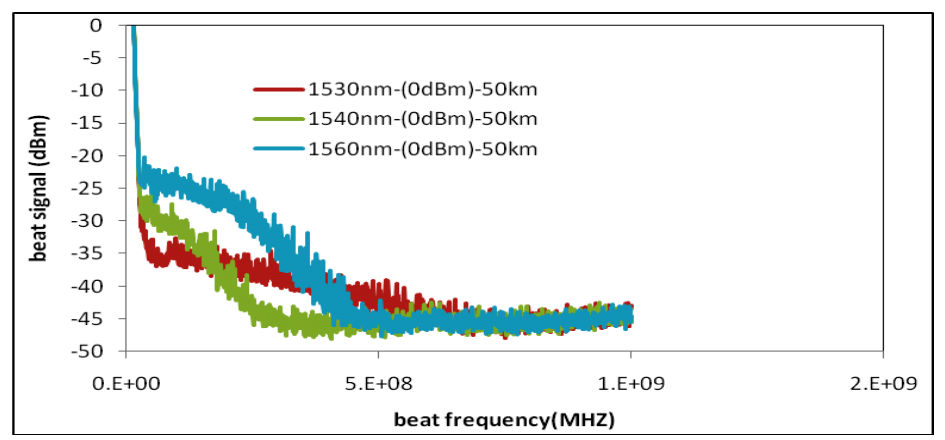

Fig. 6: The beat signal curves against beat frequency at different wavelengths which were obtained using 25 $\mathrm{km}$ 


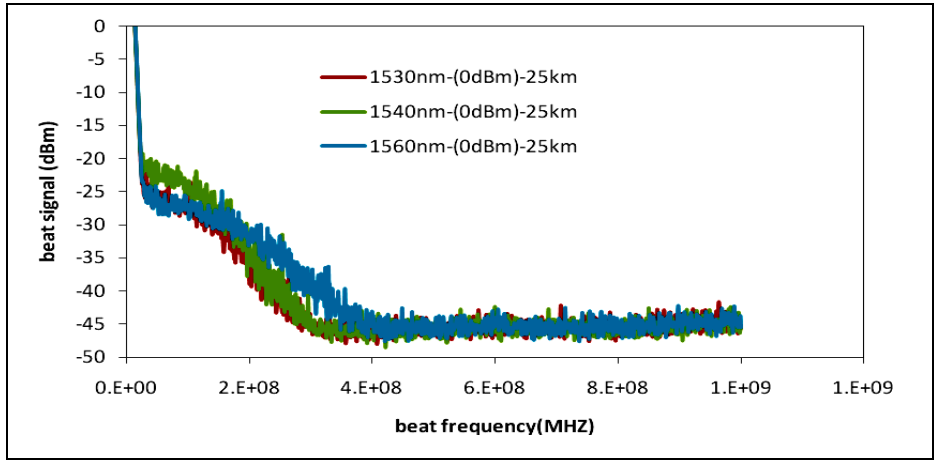

Fig. 7: The beat signal curves against beat frequency at different wavelengths which were obtained using $50 \mathrm{~km}$

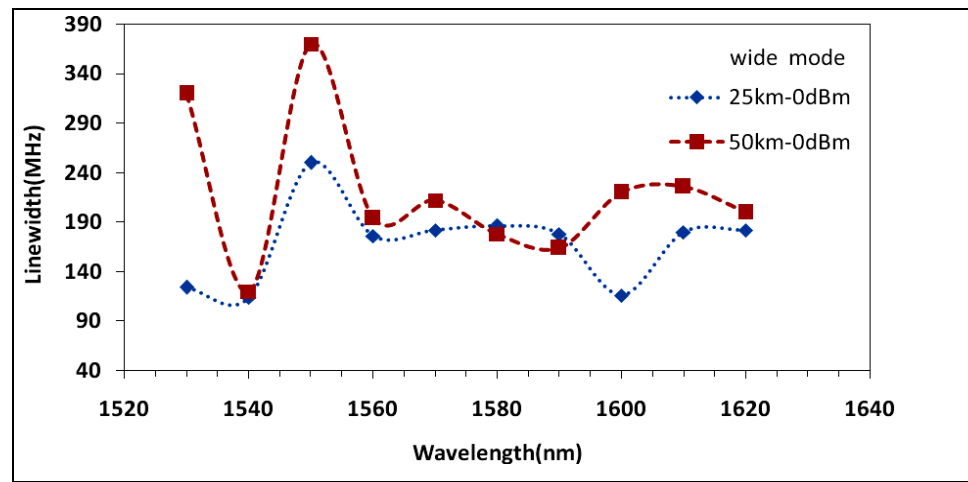

Fig 8: Relationship between linewidth and operating wavelength

\section{Heterodyne technique for linewidth measurement}

The ultra-narrow linewidth measurement is a challenge because the self- homodyne or self-heterodyne techniques require long delaying fibers which is impractical for fiber lasers due to propagation losses. The standard spectrum analyzer also has a very large dark area of more than $20 \mathrm{MHz}$, which is impractical to measure a very narrow linewidth laser. However, the laser linewidth measurement can also be done by evaluating the beat signal resulting from the interference of the laser with another uncorrelated laser [6][1][7]. This measurement technique is known as heterodyne method. This method requires another laser either with a comparable well-known spectrum or with an extremely narrow and ignorable linewidth.

Fig. 9 shows the experimental set, which consists of two laser sources (TLS 1 and TLS 2), two isolators, a PC, a $3 \mathrm{~dB}$ coupler, detector and RFSA. Optical isolator is used to avoid the backward reflection from damaging the TLS. PC is used to adjust the polarization state of one of the light source.

Fig. 10 shows the beat signal from the RF spectrum analyzer when both TLS settings are set as narrow mode. In the experiment, the input signal power and wavelength for both TLSs are set at $0 \mathrm{dBm}$ and $1550 \mathrm{~nm}$, respectively. Many beat frequency signals are observed due to the fluctuation in the TLS's wavelength. The linewidth of the light source is estimated to be around $6 \mathrm{MHz}$ as shown in the inset of Fig. 10. Fig. 11 shows the linewidth spectrum of the test signal (TLS 2) at two different linewidth settings. In the experiment, the reference source (TLS 1) is set with narrow mode. Fig. 11 (a) and (b) shows the spectrum when the TLS 2 is set with narrow and wide modes, respectively. As shown in both figures, the linewidth of $7 \mathrm{MHz}$ and $58 \mathrm{MHz}$ are obtained with the narrow and wide modes, respectively.

Fig. 12 summarizes the relationship between the linewidth value and the signal wavelength for two different modes of TLS 2. As shown in the figure, the linewidth value is smaller at the longer wavelength for both mode settings. In average, the linewidth of the TLS is obtained at around $10 \mathrm{MHz}$ and $150 \mathrm{MHz}$ for narrow and wide modes, respectively as shown in Fig. 12. Compared to the previous homodyne technique, the linewidth value obtained for wide mode is almost the same. This proves the accurac0y of this technique. 


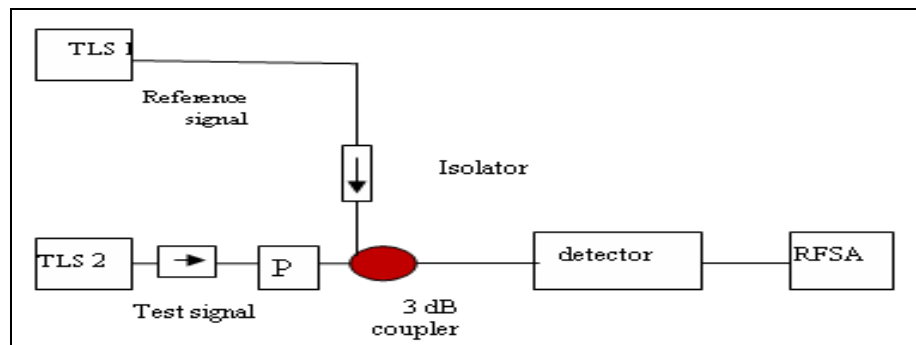

Fig. 9: Configuration of heterodyne linewidth measurement

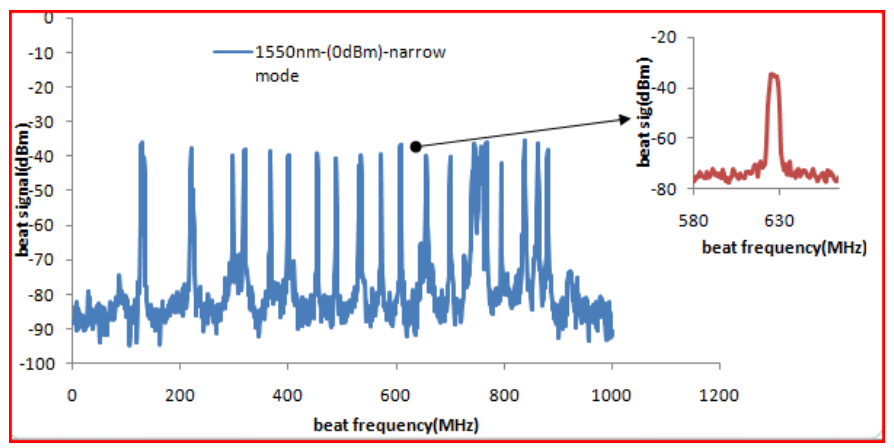

Fig. 10: Beat frequency signals from RFSA. Inset shows the enlarged picture at beat frequency of $639 \mathrm{MHz}$.

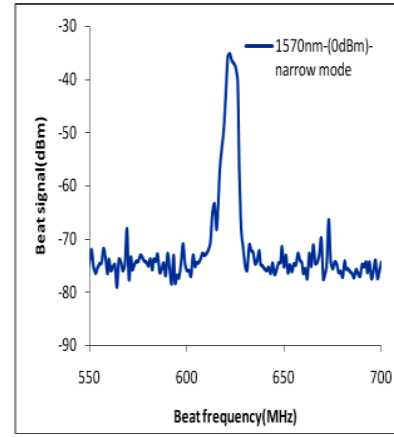

(a) Narrow mode

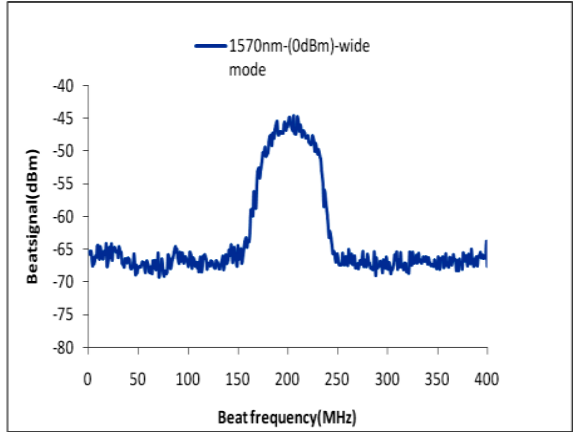

(b) Wide mode

Fig. 11: Linewidth spectrum of the test signal (TLS 2) at two different linewidth settings

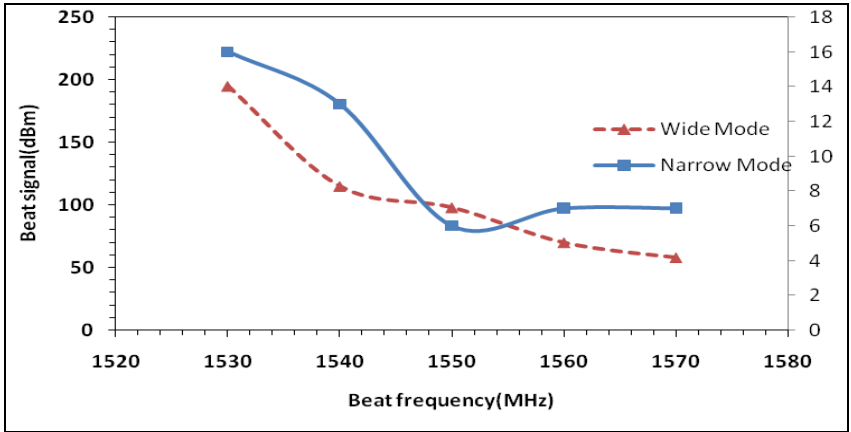

Fig. 12: Linewidth against wavelength for two different modes of TLS 2

\section{Conclusions}

Lasers with very narrow linewidth are required for various applications. The homodyne and heterodyne measurement techniques are the most established method for measuring the linewidth of a laser. In the homodyne measurement, With $25 \mathrm{~km}$ of SMF as a delay fiber, the linewidths of $124 \mathrm{MHz}, 114 \mathrm{MHz}$ and 176 $\mathrm{MHz}$ are obtained at operating wavelengths of $1530 \mathrm{~nm}, 1540 \mathrm{~nm}$ and $1560 \mathrm{~nm}$, respectively . In the experiment, the TLS setting is set at 'wide mode' and the values of the linewidth are obtained at $3 \mathrm{~dB}$ from the peak signal. At $50 \mathrm{~km} \mathrm{SMF}$, the linewidths of $320 \mathrm{MHz}, 120 \mathrm{MHz}$ and $194 \mathrm{MHz}$ are obtained at operating wavelengths of $1530 \mathrm{~nm}, 1540 \mathrm{~nm}$ and $1560 \mathrm{~nm}$, respectively. This result shows that a slightly higher value is 
obtained with a longer length of the delay fiber. The linewidths of $180 \mathrm{MHz}, 210 \mathrm{MHz}$ and $220 \mathrm{MHz}$ are obtained at operating power of $(0 \mathrm{dBm}),(-4 \mathrm{dBm})$, and $(-8 \mathrm{dBm})$, respectively. This result shows the linewidth value increases with the reduction of signal power.

The ultra-narrow linewidth measurement is a challenge because the homodyne technique requires long delaying fibers which is impractical for fiber lasers due to propagation losses. However, the laser linewidth measurement can also be done by a heterodyne method. In this method, the linewidth is evaluated from the beat signal resulting from the interference of the laser with another uncorrelated laser. In average, the linewidth of the TLS is obtained at around $10 \mathrm{MHz}$ and $150 \mathrm{MHz}$ for narrow and wide modes, respectively. The value obtained is comparable to the result obtained in the previous homodyne technique for wide mode. This proves the accuracy of this technique.

\section{References}

[1] Adnan H. Ali " Simultaneous Measurements for Tunable Laser Source Linewidth with Homodyne Detection" Computer and Information Science, Vol. 4, No. 4; July 2011.

[2] H. Ludvigsen, M. Tossavainen, and M. Kaivola, "Laser linewidth measurements using self-homodyne detection with short delay," Opt. Commun. 155(1-3), 180-186 (1998).

[3] P. Sevillano, J. Subías, C. Heras, J. Pelayo, and F. Villuendas, "Brillouin induced self-heterodyne technique for narrow line width measurement" Optics Express, Vol. 18, Issue 14, pp. 15201-15206 (2010)

[4] E. Ip, A. P. Lau, D. J. Barros, and J. M. Kahn, "Coherent detection in optical fiber systems," Opt. Express 16(2), 753-791 (2008)

[5] D. Guyomarc'h, G. Hagel, C. Zumsteg, and M. Knoop, "Some aspects of simulation and realization of an optical reference cavity," Phys. Rev. A 80, 063820 (2009).

[6] P. Horak and W. H. Loh, "On the delayed self-heterodyne interferometric technique for determining the linewidth of fiber lasers," Opt. Express 14(9), 3923-3928 (2006).

[7] Derickson, D., Fiber optic test and measurement. Upper Saddle River, N.J: 1998, Prentice Hall PTR

[8] Svelto, O., Principles of Lasers, Plenum Press, New York and London, 4th ed, 1998. 